|||III||||||||||||||||||

ACF-Kids (063)

PIt 1 (001)
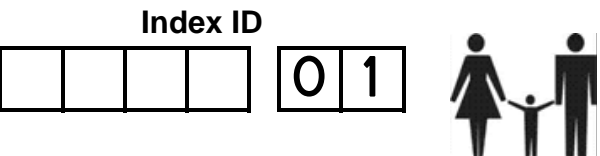

Index Child's

Interview
Visit 1 (010)

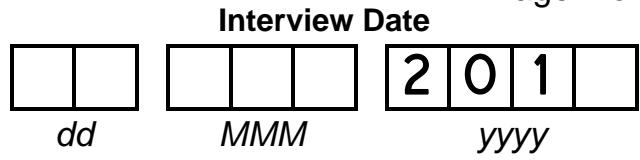

Interviewer Initials:

Consent obtained from caregiver?........ $\square$ Yes $\square$ No

\title{
Index Case Details:
}

1. Sex:

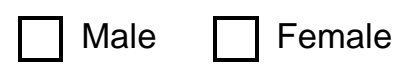

2. Date of birth:
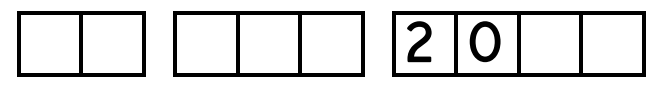

(dd/MMM/yyyy)

3. Child is:

Outpatient $\square$ Inpatient

\section{Sociodemographic Information:}

4. How many years has the child lived in this house?

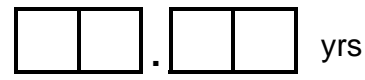

5. How many people reside in the child's house?

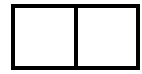

6. Estimate of combined household income of the child's house?

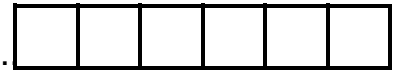
$\mathrm{R} / \mathrm{mth}$

7. Are there any smokers in the child's house?. $\square$ Yes $\square$ No

7a. If Yes to Q7, do they smoke indoors? $\square$ Yes $\square$ No

8. Are there any person(s) living in the child's house that have been to prison? $\square$ Yes $\square$ No $\rightarrow$ If No, go to Q9

8a. How many months did that person spend in prison?

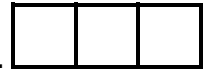

$8 \mathrm{~b}$. When was the person last in prison?

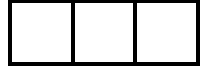

MMM

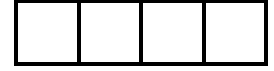

yyyy

8c. Is that person coughing now?

$\square$ Yes $\square$ No

8d. Has that person been diagnosed with TB?

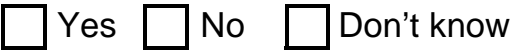

9. Does anyone in the child's house currently have TB, or had TB in the past two years?.. $\square$ Yes $\square$ No $\rightarrow$ If No, go

If Yes to Q9, how many people?

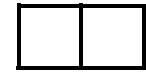

(Complete relevant lines below)
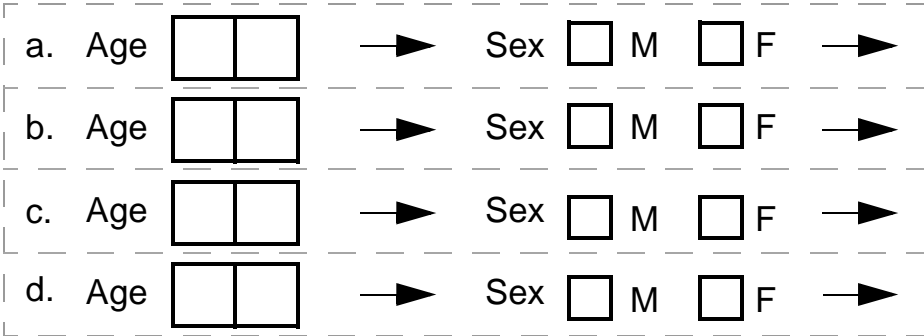

Number of previous TB episodes before 2008

Number of previous TB episodes before 2008

Number of previous TB episodes before 2008

Number of previous TB episodes before 2008

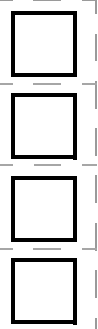

10. Index child's height/length:

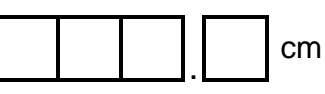

11. Index child's weight:

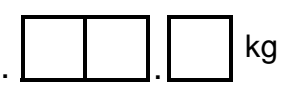




\section{Active Case Findings - ACF Kids}

Please Initial and date the appropriate section below:

1st Review:

2nd Review: $\quad$ Initials $-\frac{1}{\text { Date }} / 20 \quad$ Faxed by: $\_$Initials $-\frac{1}{\text { Date }} / 20$

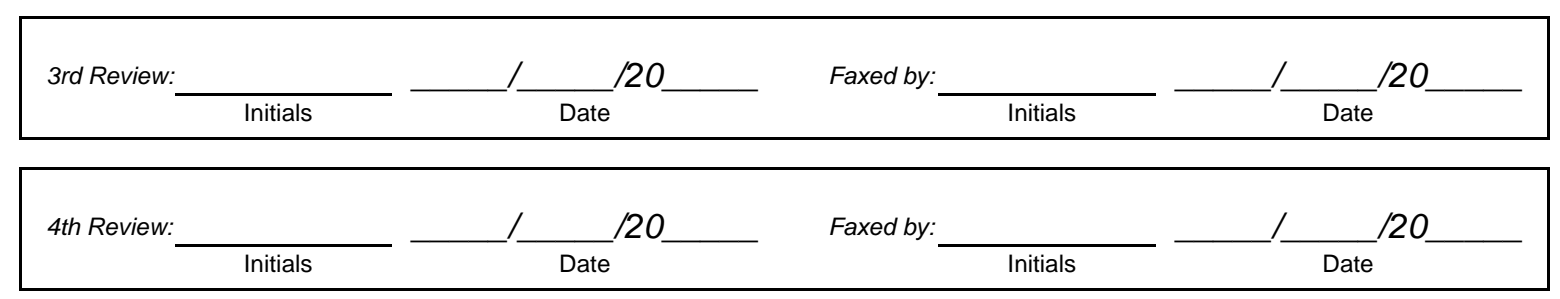


| | IIIIII| | | | | | | | || | | | | | | | | | |

ACF-Kids (063)

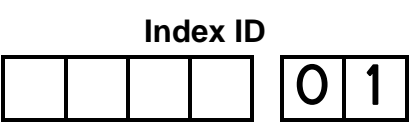

PIt 2 (002)

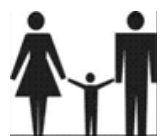

Visit 1 (010)

Index Child's

Interview

Page 2 of 4

\section{History of current illness:}

12. What was the start date of the symptoms that brought the child to hospital?.
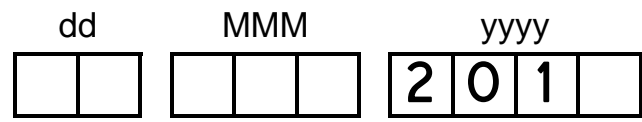

13. What are Index child's current symptoms?... Yes

Cough.

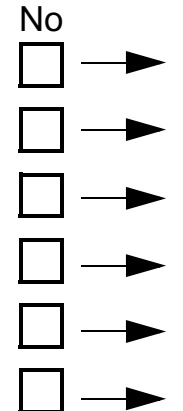

If yes, duration:.

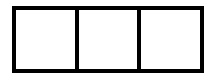

Weight loss.

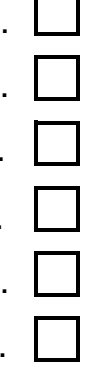

If yes, duration:.

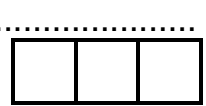

Fever.

If yes, duration:

If yes, duration:.

Lethargy.

Night sweats

If yes, duration:.

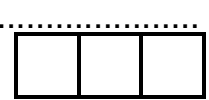

days

Loss of appetite.

If yes, duration:

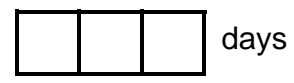

days

TB History and risk:

Yes No

14. Has the child had TB previously?

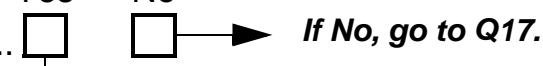

If Yes, number of episodes?

15. What was the date of diagnosis of your child's most recent TB episode?

(Before current admission)

Yes No
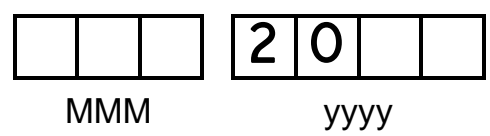

16. Did child complete full TB treatment?

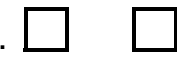

Yes No

If Yes, how long has that

17. Is there any person living with this child who is coughing?

19. Date of discharge or death of the child?

a.

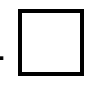

20. Diagnosis (ICD-10)?

Ask Dr Moor or Dr Lala for assistance and draw a line through the UNUSED diagnoses fields.

b.

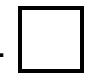

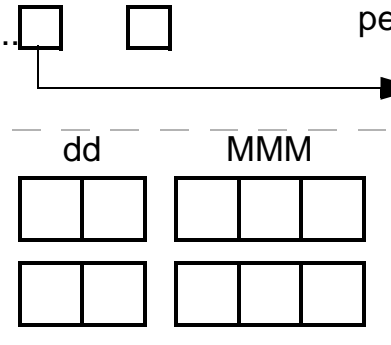

C.

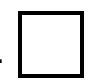

d.

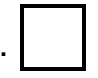

e.
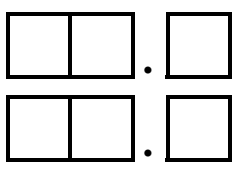
person been coughing?
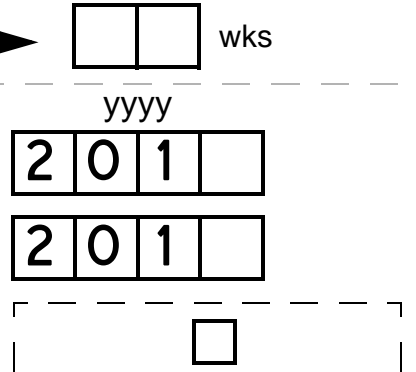

Check this box if child died in hospital.
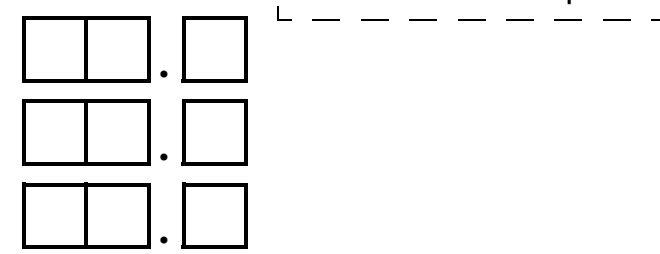

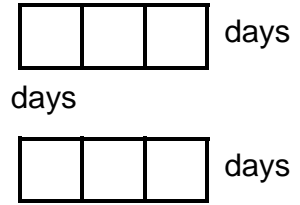

21. HIV status of mother at the time of this child's birth?

HIV infected

$\square$ HIV uninfected

HIV unknown

22. HIV status of this child? $\square$ HIV infected

$\square$ HIV uninfected

22a. Child receiving Antiretroviral therapy?

$\square$ Yes $\square$ No

i. Date ARVs started?

ii. List ARV codes below:
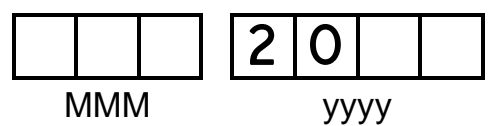

a.

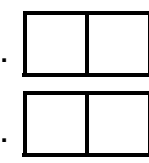

b.

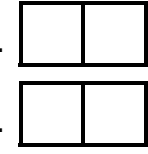

c.

f.
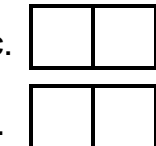


\section{Active Case Findings - ACF Kids}

Please Initial and date the appropriate section below:

1st Review:

2nd Review: $\quad$ Initials $-\frac{1}{\text { Date }} / 20 \quad$ Faxed by: $\_$Initials $-\frac{1}{\text { Date }} / 20$

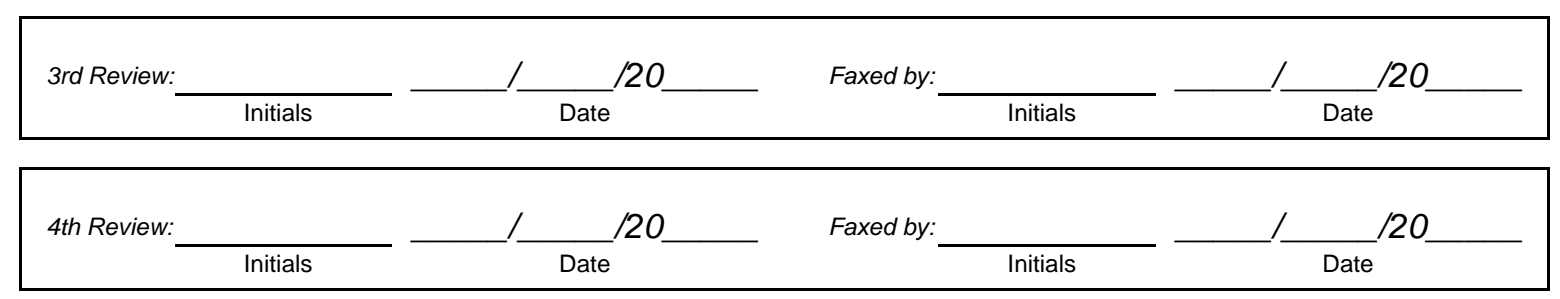


| |||||||||||||||||||||||||

$\begin{array}{lll}\text { ACF-Kids (063) Plt } 3(003) & \text { Visit } 1(010)\end{array}$

\begin{tabular}{|l|l|}
\hline 0 & 1 \\
\hline
\end{tabular}

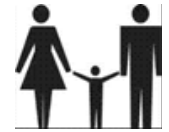

Index Child's

Interview

23. Child's chest X-ray?.

$\square$ Done $\square$ Not done
rate/Consolidation $\square$ Miliary pattern
itation
$\square$ Other:

24. Abdominal sonar?

$\square$ Not done

$\square$ Normal

Splenic microabscesses

$\square$ Lymphadenopathy $\square$ Other:

25. Tuberculosis skin test (TST/PPD) at this admission?

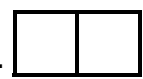
$\mathrm{mm}$ (99 if not done)

26. Date sputum specimen collected:
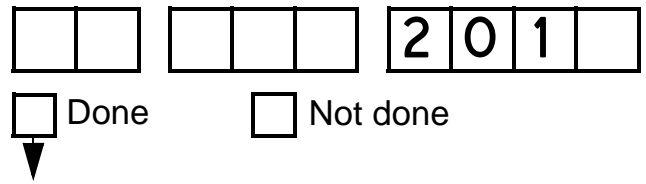

(dd/MMM/yyyy)

27. Sputum smear (AFB):

(If any positive, use the highest count.)

\begin{tabular}{|c|c|c|}
\hline \multirow{2}{*}{ Result: } & Negative/Not seen & Moderate $(++)$ \\
\hline & Scanty (+) & Numerous $(+++)$ \\
\hline
\end{tabular}

28. Culture:

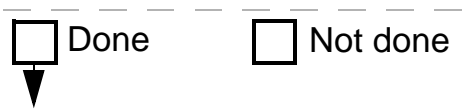

28a. Result: ZN for AAFB: $\square$ Positive $\square$ Negative $\square$ Unknown

28b. Number of days to positive:

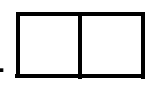

28c. ID of Mycobacterium:

M Tuberculosis

$\square$ Other

M Avium Complex

Contaminated / Lab problem

28d. Drug sensitivity: $\square$ Done $\square$ Not Done

If resistant to Isoniazid AND Rifampacin

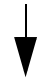

Resistant to 2nd line drugs?

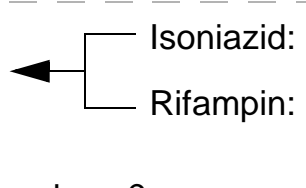

$\square$ Resistant

$\square$ Sensitive

$\square$ Not done

(If Yes, specify which drugs below)

$\square$ Resistant $\square$ Sensitive $\square$ Not done Yes

$\square$ No

a.

b.

29. Other biological specimen:

(e.g. FNA, biopsy, gastric aspirate)

$\square$ Collected $\square$ Not collected

29a. Specify:

29b. Date obtained:
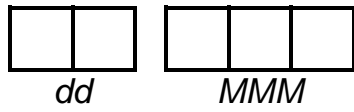

-

Page 3 of 4 


\section{Active Case Findings - ACF Kids}

Please Initial and date the appropriate section below:

1st Review:

2nd Review: $\quad$ Initials $-\frac{1}{\text { Date }} / 20 \quad$ Faxed by: $\_$Initials $-\frac{1}{\text { Date }} / 20$

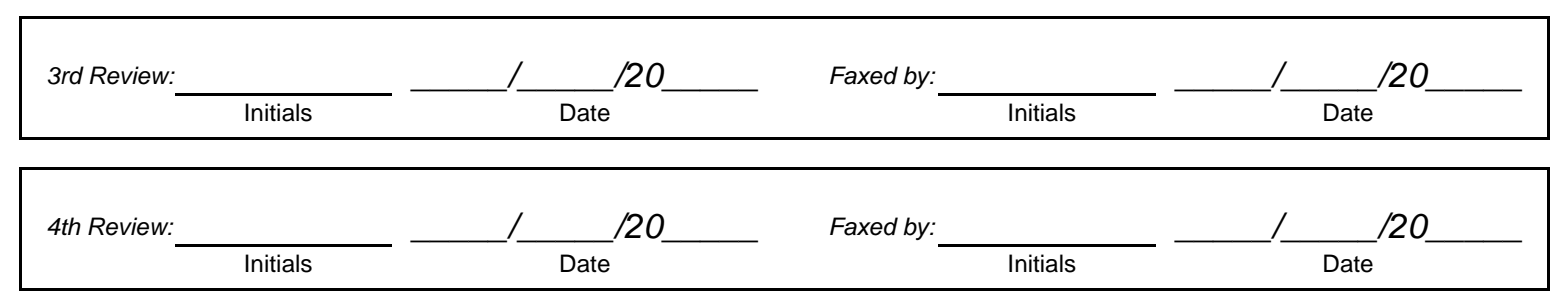




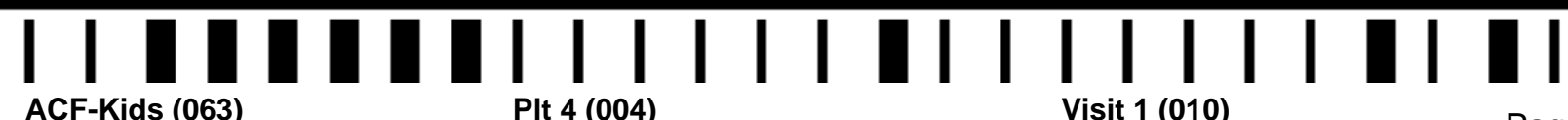

ACF-Kids (063)

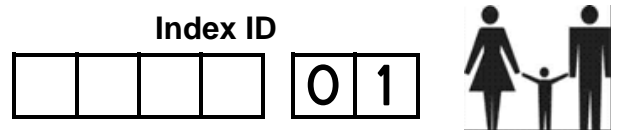

Index Child's

Interview

30. Child on TB treatment?

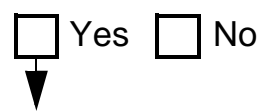

30a. Date TB treatment started?..
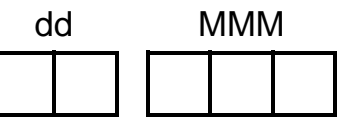

\begin{tabular}{|l|l|l|l|}
\multicolumn{4}{c}{ yyyy } \\
\hline 2 & 0 & 1 & \\
\hline
\end{tabular}

30b. Regimen?

1st episode

$\square$ 2nd episode

TBM

MDR/XDR

"Liver friendly"

31. Pyridoxine given to child?......... $\square$ Yes $\square$ No

32. Prednisone given to child?....... $\square$ Yes $\square$ No 


\section{Active Case Findings - ACF Kids}

Please Initial and date the appropriate section below:

1st Review:

2nd Review: $\quad$ Initials $-\frac{1}{\text { Date }} / 20 \quad$ Faxed by: $\_$Initials $-\frac{1}{\text { Date }} / 20$

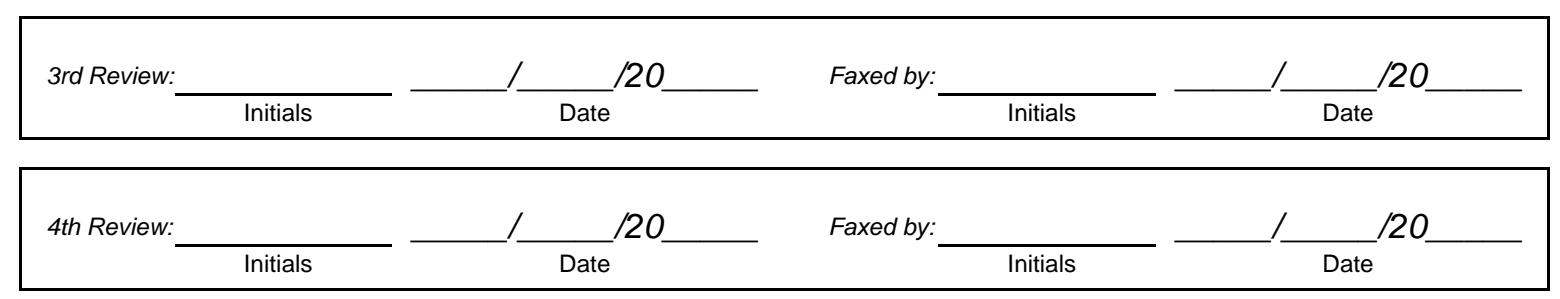

